\title{
OPEN Changes in retinal microvasculature and retinal layer thickness in association with apolipoprotein E genotype in Alzheimer's disease
}

\author{
Joo Youn Shin ${ }^{1}$, Eun Young Choi ${ }^{2}$, Min Kim ${ }^{\boxplus}$, Hyung Keun Lee ${ }^{2}$ \& Suk Ho Byeon ${ }^{1}$
}

Biomarker tests of Alzheimer's disease (AD) are invasive and expensive. Recent developments in optical coherence tomography (OCT) and OCT angiography (OCTA) have enabled noninvasive, cost-effective characterization of retinal layer vasculature and thickness. Using OCTA and OCT, we characterized retinal microvascular changes in the mild cognitive impairment (MCI) stage of AD and assessed their correlation with structural changes in each retinal neuronal layer. We also evaluated the effect of the $A P O E-\varepsilon 4$ genotype on retinal microvasculature and layer thickness. Retinal layer thickness did not differ between $\mathrm{MCl}$ patients ( 40 eyes) and controls ( 37 eyes, all $\mathrm{p}>0.05$ ). $\mathrm{MCl}$ patients had lower vessel density (VD) $(p=0.003)$ of the superficial capillary plexus $(S C P)$ and larger foveal avascular zone area $(p=0.01)$ of the deep capillary plexus (DCP) than those of controls. VD of the SCP correlated with the ganglion cell layer $(r=0.358, p=0.03)$ and inner plexiform layer thickness $(r=0.437$, $\mathrm{p}=0.007$ ) in $\mathrm{MCl}$ patients. APOE- $\varepsilon 4$-carrying $\mathrm{MCl}$ patients had a lower VD of the DCP than non-carriers $(p=0.03)$. In conclusion, retinal microvasculature was reduced in patients with $A D$-associated $M C l$, but retinal thickness was not changed; these changes might be affected by the APOE genotype. OCTA of the retinal microvasculature may be useful to detect vascular changes in AD.

Alzheimer's disease (AD) is a progressive neurodegenerative disease characterized by a gradual decline in memory and cognitive function. The main pathological features are the deposition of $A \beta$-peptide and hyperphosphorylation of tau protein; however, vascular factors are also considered to be involved in the pathophysiology of $\mathrm{AD}^{1}$. Although environmental factors may affect the risk of sporadic $\mathrm{AD}$, studies in twins have suggested that genetic factors play a critical role in late-onset $\mathrm{AD}^{2}$. Among the many susceptibility genes for $\mathrm{AD}$, the $\varepsilon 4$ allele of apolipoprotein $\mathrm{E}(A P O E-\varepsilon 4)$ is the major genetic risk factor for both early- and late-onset $\mathrm{AD}^{3}$. $A P O E-\varepsilon 4$ carriers were reported to have an increased risk of $\mathrm{AD}$ (3-15 times) in a gene dose-dependent manner ${ }^{4}$; these carriers showed different treatment responses to amyloid removal therapy and had more vasogenic edema ${ }^{5}$, suggesting a different mechanism of action of $A P O E-\varepsilon 4$ in the pathogenesis of $A D$.

Currently available treatments for $\mathrm{AD}$ aim to maximize the remaining activity of the neurons affected by the disease, although they cannot slow down its progression. Thus, there is a great interest in finding biomarkers to screen individuals in the earlier stage of the disease, including those with mild cognitive impairment (MCI) or early $\mathrm{AD}$ who would benefit from potential therapy. Current $\mathrm{AD}$ biomarker tests mainly include positron emission tomography (PET) or cerebrospinal fluid testing, which is highly invasive and expensive. Noninvasive, technically simple, and inexpensive biomarkers are needed for diagnosis or therapeutic monitoring of AD in the clinical setting or population-wide screening ${ }^{6}$.

The retina shares similar anatomical and physiological features with the brain; numerous studies have reported changes in the retina in patients with $\mathrm{AD}$, suggesting that the retina can be a possible biomarker for diagnosing, screening, and managing $\mathrm{AD}$ in clinical practice ${ }^{7}$. The retina can be noninvasively assessed using high-resolution images obtained with optical coherence tomography (OCT). Thinning of the retinal nerve fiber layer (RNFL) and ganglion cell-inner plexiform layer (GC-IPL), and decreased macular volume have been reported to be associated with $\mathrm{AD}^{7,8}$. A recent study reported that a thinner RNFL at baseline indicates an increased risk of dementia and

${ }^{1}$ Department of Ophthalmology, Institute of Vision Research, Severance Hospital, Yonsei University College of Medicine, Seoul, Republic of Korea. ${ }^{2}$ Department of Ophthalmology, Institute of Vision Research, Gangnam Severance Hospital, Yonsei University College of Medicine, Seoul, Republic of Korea. ${ }^{\bowtie}$ email: minkim76@ gmail.com 


\begin{tabular}{|l|l|l|c|}
\hline & MCI $(\mathbf{n}=\mathbf{4 0})$ & Controls $(\mathbf{n}=\mathbf{3 7})$ & p-value \\
\hline Age, mean \pm SD, years & $72.8 \pm 8.6$ & $69.0 \pm 10.4$ & 0.15 \\
\hline Sex $(\mathrm{M} / \mathrm{F}),(\mathrm{n} / \mathrm{n})$ & $25 / 15$ & $17 / 20$ & 0.17 \\
\hline DM $(\mathrm{n})$ & 3 & 6 & 0.30 \\
\hline HTN $(\mathrm{n})$ & 9 & 14 & 0.21 \\
\hline OD/OS (n/n) & $20 / 20$ & $19 / 18$ & $>0.999$ \\
\hline SE, mean \pm SD, Diopter & $-0.48 \pm 2.1$ & $-0.45 \pm 1.8$ & 0.84 \\
\hline VA, mean \pm SD, logMAR & $0.03 \pm 0.05$ & $0.04 \pm 1.8$ & 0.28 \\
\hline Retinal neuronal layer thickness, mean \pm SD, $\mu \mathrm{m}$ & & \\
\hline Total retina & $315.1 \pm 14.2$ & $314.9 \pm 14.4$ & 0.87 \\
\hline RNFL & $20.0 \pm 2.4$ & $19.9 \pm 2.5$ & 0.95 \\
\hline GCL & $39.1 \pm 3.8$ & $38.3 \pm 6.3$ & 0.92 \\
\hline IPL & $34.6 \pm 2.1$ & $33.9 \pm 3.6$ & 0.47 \\
\hline INL & $37.2 \pm 3.9$ & $36.1 \pm 3.3$ & 0.20 \\
\hline OPL & $33.7 \pm 4.7$ & $33.5 \pm 5.1$ & 0.83 \\
\hline ONL & $67.5 \pm 9.9$ & $68.3 \pm 8.5$ & 0.96 \\
\hline RPE & $15.3 \pm 1.7$ & $16.0 \pm 1.9$ & 0.25 \\
\hline
\end{tabular}

Table 1. Demographic characteristics and retinal neuronal layer thickness of the participants. $\mathrm{MCI}=$ mild cognitive impairment, $\mathrm{M}=$ male, $\mathrm{F}=$ female, $\mathrm{DM}$ = diabetes mellitus, $\mathrm{HTN}$ = hypertension, $\mathrm{SE}=$ spherical equivalent, $\mathrm{OD}=$ right eye, $\mathrm{OS}=$ left eye, $\mathrm{VA}=$ visual acuity, $\log \mathrm{MAR}=\operatorname{logarithm}$ of the minimum angle of resolution, $\mathrm{RNFL}=$ retinal nerve fiber layer, $\mathrm{GCL}=$ ganglion cell layer, $\mathrm{IPL}=$ inner plexiform layer, $\mathrm{INL}=\mathrm{inner}$ nuclear layer, $\mathrm{OPL}=$ outer plexiform layer, $\mathrm{ONL}=$ outer nuclear layer, $\mathrm{RPE}=$ retinal pigment epithelium, $\mathrm{SD}=$ standard deviation. ${ }^{*} \mathrm{p}<0.05$.

AD over the follow-up period, suggesting that OCT measurements of the retina can help identify individuals at a high risk of very early cognitive changes and can help develop better clinical trials ${ }^{9,10}$.

Recent developments in OCT angiography (OCTA) have enabled the characterization of the vasculature in retinal layers at the micrometer level, providing a quantitative assessment of the microvascular structure in the retina. A few studies have reported changes in the microvasculature of AD patients using OCTA, suggesting the presence of retinal microvascular dysfunction ${ }^{11-15}$; however, little is known about the relationship between changes in the retinal microvasculature and retinal neuronal layers and the $A P O E-\varepsilon 4$ genotype. In the current study, we aimed to characterize retinal microvascular changes that occur in the MCI stage of AD using OCTA and assess their correlation with retinal structural changes in each retinal neuronal layer. In addition, we evaluated the effect of the $A P O E-\varepsilon 4$ genotype on retinal microvasculature and retinal layer thickness.

\section{Results}

Baseline characteristics and thickness of each retinal layer. A total of 40 eyes from 24 patients with $\mathrm{MCI}$ owing to $\mathrm{AD}$ and 37 eyes from 31 control subjects were analyzed. All participants were Asians (Korean) and had a global Clinical Dementia Rating (CDR) score of 0.5 and a Mini-Mental State Examination (MMSE) score of $>24$. The baseline characteristics of the MCI and control groups are shown in Table 1 . There were no statistically significant differences in terms of age $(\mathrm{p}=0.15)$, sex $(\mathrm{p}=0.17)$, spherical equivalent $(S E ; \mathrm{p}=0.84)$, visual acuity $(\mathrm{p}=0.28)$, and presence of diabetes mellitus $(\mathrm{p}=0.30)$ and hypertension $(\mathrm{p}=0.21)$ between the two groups. No significant differences were found in total retinal thickness $(\mathrm{p}=0.87)$ and thickness of each retinal layer between the MCI and control groups.

Comparison of OCTA parameters between $\mathrm{MCI}$ patients and controls. In the superficial capillary plexus (SCP), significantly lower vessel density (VD) was observed in the MCI group than in the control group $(p=0.003)$, whereas there was no significant difference in the foveal avascular zone (FAZ) area $(p=0.17)$. In the deep capillary plexus $(\mathrm{DCP})$, there was no difference in VD $(\mathrm{p}=0.80)$ between the MCI and control groups; however, a larger FAZ area was observed in the MCI group than in the control group $(p=0.011)$ (Table 2).

The comparison of VDs of the SCP in each subfield between the two groups revealed that the MCI group had a significantly lower density than the control group in the whole subfield, except for the nasal inner $(p=0.25)$ and outer subfields $(\mathrm{p}=0.53)$ (Table 3$)$.

Correlation of VD with the thickness of each retinal layer. In the MCI group, the VD of the SCP was significantly correlated with ganglion cell layer $(\mathrm{GCL})$ thickness $(\mathrm{p}=0.03)$ and inner plexiform layer (IPL) thickness $(p=0.007)$, whereas the VD of the DCP showed no correlation with the thickness of all retinal neuronal layers. Meanwhile, there was no significant correlation between VD and thickness of each retinal layer in both SCP and DCP in the control group (Table 4).

Comparison of OCT and OCTA parameters between APOE- $\varepsilon 4$ carriers and non-carriers. The $A P O E$ genotype was evaluated in $16 \mathrm{MCI}$ patients, of whom six (10 eyes) were $A P O E-\varepsilon 4$ carriers, and 10 (18 


\begin{tabular}{|c|c|c|c|}
\hline & $\operatorname{MCI}(n=40)$ & Controls $(n=37)$ & p-value \\
\hline \multicolumn{4}{|c|}{$\mathrm{SCP}$, mean \pm SD } \\
\hline $\mathrm{FAZ}\left(\mathrm{mm}^{2}\right)$ & $0.31 \pm 0.11$ & $0.27 \pm 0.09$ & 0.17 \\
\hline VD (\%) & $14.0 \pm 3.9$ & $16.3 \pm 2.5$ & $0.003^{*}$ \\
\hline \multicolumn{4}{|c|}{$\mathrm{DCP}$, mean \pm SD } \\
\hline FAZ $\left(\mathrm{mm}^{2}\right)$ & $0.95 \pm 0.24$ & $0.80 \pm 0.20$ & $0.011^{\star}$ \\
\hline VD (\%) & $25.5 \pm 1.9$ & $25.6 \pm 1.8$ & 0.80 \\
\hline
\end{tabular}

Table 2. Comparison of optical coherence tomography angiography parameters between patients with mild cognitive impairment and controls. $\mathrm{MCI}=$ mild cognitive impairment, $\mathrm{SCP}=$ superficial capillary plexus, $\mathrm{FAZ}=$ foveal avascular zone, $\mathrm{VD}=$ vessel density, $\mathrm{DCP}=$ deep capillary plexus, $\mathrm{SD}=$ standard deviation. ${ }^{*} \mathrm{p}<0.05$.

\begin{tabular}{|l|l|l|l|c|}
\hline Subfield, mean \pm SD & MCI $(\mathbf{n}=\mathbf{4 0})$ & Controls $(\mathbf{n}=\mathbf{3 7})$ & Raw $\mathbf{p}$-value & Benjamini-Hochberg p-value \\
\hline Central (\%) & $5.63 \pm 3.9$ & $7.8 \pm 3.4$ & $0.007^{*}$ & $0.013^{\star}$ \\
\hline Inner superior (\%) & $14.2 \pm 4.4$ & $16.5 \pm 3.3$ & $0.012^{\star}$ & $0.018^{\star}$ \\
\hline Inner nasal (\%) & $14.1 \pm 4.7$ & $15.5 \pm 3.8$ & 0.22 & 0.248 \\
\hline Inner inferior (\%) & $14.2 \pm 4.6$ & $16.1 \pm 3.9$ & $0.032^{\star}$ & $0.041^{\star}$ \\
\hline Inner temporal (\%) & $14.2 \pm 4.4$ & $16.8 \pm 3.1$ & $0.002^{*}$ & $0.009^{*}$ \\
\hline Outer superior (\%) & $14.4 \pm 4.4$ & $17.0 \pm 2.4$ & $0.006^{\star}$ & $0.013^{\star}$ \\
\hline Outer nasal (\%) & $15.9 \pm 4.7$ & $16.9 \pm 3.4$ & 0.53 & 0.53 \\
\hline Outer inferior (\%) & $13.8 \pm 4.4$ & $16.5 \pm 2.4$ & $0.003^{\star}$ & $0.009^{\star}$ \\
\hline Outer temporal (\%) & $13.2 \pm 4.4$ & $16.7 \pm 3.3$ & $<0.001^{\star}$ & $<0.001^{*}$ \\
\hline
\end{tabular}

Table 3. Comparison of the vessel densities of the superficial capillary plexus in each subfield between the groups. $\mathrm{MCI}=$ mild cognitive impairment, $\mathrm{SD}=$ standard deviation. ${ }^{\star} \mathrm{p}<0.05$.

\begin{tabular}{|c|c|c|c|c|}
\hline & \multicolumn{2}{|l|}{ MCI } & \multicolumn{2}{|l|}{ Controls } \\
\hline & Spearman's correlation coefficient & p-value & Spearman's correlation coefficient & p-value \\
\hline \multicolumn{5}{|c|}{ Correlation with VD of the SCP } \\
\hline Total retina & 0.224 & 0.18 & 0.125 & 0.52 \\
\hline RNFL & -0.220 & 0.19 & -0.122 & 0.53 \\
\hline GCL & 0.358 & $0.030^{*}$ & 0.120 & 0.54 \\
\hline IPL & 0.437 & $0.007^{*}$ & 0.170 & 0.38 \\
\hline INL & 0.089 & 0.60 & -0.073 & 0.71 \\
\hline OPL & 0.133 & 0.43 & -0.379 & 0.053 \\
\hline ONL & 0.051 & 0.76 & 0.335 & 0.076 \\
\hline RPE & 0.141 & 0.40 & 0.167 & 0.39 \\
\hline \multicolumn{5}{|c|}{ Correlation with VD of the DCP } \\
\hline Total retina & -0.101 & 0.55 & 0.427 & 0.20 \\
\hline RNFL & -0.280 & 0.09 & 0.218 & 0.26 \\
\hline GCL & 0.118 & 0.49 & 0.095 & 0.62 \\
\hline IPL & -0.131 & 0.44 & 0.223 & 0.24 \\
\hline INL & -0.234 & 0.16 & 0.382 & 0.40 \\
\hline OPL & -0.118 & 0.49 & 0.085 & 0.66 \\
\hline ONL & 0.115 & 0.50 & 0.179 & 0.35 \\
\hline RPE & 0.056 & 0.74 & -0.133 & 0.49 \\
\hline
\end{tabular}

Table 4. Correlation of vessel density with each retinal neuronal layer thickness in the two groups. MCI = mild cognitive impairment, $\mathrm{VD}=$ vessel density, $\mathrm{SCP}=$ superficial capillary plexus, $\mathrm{RNFL}=$ retinal nerve fiber layer, $\mathrm{GCL}=$ ganglion cell layer, $\mathrm{IPL}=$ inner plexiform layer, $\mathrm{INL}=$ inner nuclear layer, $\mathrm{OPL}=$ outer plexiform layer, $\mathrm{ONL}=$ outer nuclear layer, $\mathrm{RPE}=$ retinal pigment epithelium, $\mathrm{DCP}=$ deep capillary plexus. ${ }^{*} \mathrm{p}<0.05$. 


\begin{tabular}{|c|c|c|c|}
\hline & APOE- $\varepsilon 4+$ & APOE- $\varepsilon 4-$ & p-value \\
\hline $\mathrm{n}$ & 10 & 18 & \\
\hline Age, mean $\pm S D$, years & $71.1 \pm 7.1$ & $73.2 \pm 6.5$ & 0.27 \\
\hline $\operatorname{Sex}(M / F),(n / n)$ & $5 / 5$ & $13 / 5$ & 0.41 \\
\hline \multicolumn{4}{|c|}{ Retinal microvasculature, mean \pm SD } \\
\hline FAZ area $\left(\mathrm{mm}^{2}\right)-\mathrm{SCP}$ & $0.32 \pm 0.09$ & $0.31 \pm 0.10$ & 0.65 \\
\hline FAZ area $\left(\mathrm{mm}^{2}\right)-\mathrm{DCP}$ & $1.04 \pm 0.24$ & $0.91 \pm 0.26$ & 0.21 \\
\hline VD (\%)-SCP & $14.4 \pm 3.8$ & $14.4 \pm 3.5$ & 0.83 \\
\hline VD (\%)-DCP & $24.3 \pm 1.8$ & $26.0 \pm 2.0$ & $0.03^{*}$ \\
\hline \multicolumn{4}{|c|}{ Retinal layer thickness, mean $\pm S D, \mu \mathrm{m}$} \\
\hline Total retina & $315.8 \pm 14.2$ & $316.2 \pm 13.8$ & 0.80 \\
\hline RNFL & $21.0 \pm 3.3$ & $19.8 \pm 1.8$ & 0.45 \\
\hline GCL & $39.7 \pm 4.7$ & $39.7 \pm 3.3$ & 0.60 \\
\hline IPL & $35.6 \pm 1.7$ & $34.6 \pm 1.9$ & 0.15 \\
\hline INL & $37.9 \pm 3.5$ & $36.6 \pm 4.2$ & 0.36 \\
\hline OPL & $35.2 \pm 4.9$ & $32.2 \pm 3.1$ & 0.17 \\
\hline ONL & $64.7 \pm 9.1$ & $69.3 \pm 9.5$ & 0.23 \\
\hline RPE & $14.7 \pm 1.7$ & $15.7 \pm 1.4$ & 0.07 \\
\hline
\end{tabular}

Table 5. Optical coherence tomography and optical coherence tomography angiography parameters of $A P O E-\varepsilon 4$ carriers and non-carriers. $A P O E=$ apolipoprotein $\mathrm{E}, \mathrm{M}=$ male, $\mathrm{F}=$ female, $\mathrm{FAZ}=$ foveal avascular zone, $\mathrm{SCP}=$ superficial capillary plexus, $\mathrm{DCP}=$ deep capillary plexus, $\mathrm{VD}=$ vessel density, $\mathrm{RNFL}=$ retinal nerve fiber layer, GCL = ganglion cell layer, $\mathrm{IPL}=$ inner plexiform layer, $\mathrm{INL}=$ inner nuclear layer, $\mathrm{OPL}=\mathrm{outer}$ plexiform layer, $\mathrm{ONL}=$ outer nuclear layer, $\mathrm{RPE}=$ retinal pigment epithelium, $\mathrm{SD}=$ standard deviation. ${ }^{*} \mathrm{p}<0.05$.

eyes) were non-carriers (Table 5). There was no significant difference in terms of age $(p=0.27)$, sex $(p=0.41)$, MMSE scores $(\mathrm{p}=0.56)$, and Seoul Neuropsychological Screening Battery (SNSB) scores $(\mathrm{p}=0.58)$. There was no difference in the FAZ area of the SCP $(p=0.65)$ and the FAZ area of the DCP $(p=0.21)$ between the two groups. $A P O E-\varepsilon 4$ carriers had lower VD of the DCP than non-carriers $(\mathrm{p}=0.03)$, whereas there was no difference in VD of the SCP $(\mathrm{p}=0.83)$. In terms of retinal layer thickness, no significant difference was observed in each retinal layer with regard to $A P O E-\varepsilon 4$ status.

\section{Discussion}

In the current study, patients with MCI owing to AD had lower VD of the SCP compared with controls who showed no decrease in the thickness of each retinal layer. Furthermore, lower VD was correlated with thinner GCL and IPL in MCI patients, suggesting that changes in the retinal microvasculature occur in the earlier stages of $\mathrm{AD}$, and these changes may precede the reduction of retinal thickness in patients with $\mathrm{AD}$. In addition, we demonstrated differences in the retinal microvasculature under different $A P O E-\varepsilon 4$ statuses, indicating that the $A P O E$ genotype might also affect the changes in retinal microvasculature.

$\mathrm{AD}$ is pathologically characterized by amyloid deposits and neurofibrillary tangles. Furthermore, vascular dysfunction has been reported in patients with $\mathrm{AD}^{1}$, although it is unclear whether it precedes and contributes to neural death or whether it is an incidental effect of decreased metabolic demand. Cerebral vascular impairment was recognized as one of the earliest pathologic features in $\mathrm{AD}^{16}$, and histopathological studies have shown the presence of cerebral capillary degeneration ${ }^{1,17}$. Furthermore, the accumulation of amyloid-beta deposits in the internal vessel walls was suggested to cause the occlusion of vascular structures and decrease the blood flow ${ }^{13}$. The results of recent studies using OCTA show that these vascular changes occur not only in the cerebral vasculature, but also in the retinal microvasculature in patients with AD. Decreased retinal VD has been reported in patients with $\mathrm{AD}, \mathrm{MCI}$, and preclinical $\mathrm{AD}^{11-14}$. A progressive trend of retinal microvascular loss (both in the SCP and $\mathrm{DCP}$ ) was observed because of $\mathrm{MCI}$ owing to $\mathrm{AD}$, indicating retinal vascular impairment during disease progression, which may contribute to the potential conversion from $\mathrm{MCI}$ to $\mathrm{AD}^{11}$. Our results also showed lower VD of the SCP and larger FAZ area of the DCP in MCI patients than in controls.

Several studies have reported significant retinal thinning of the GC-IPL and a reduction in macular thickness and macular volume in the eyes of AD patients ${ }^{7,8}$. The proposed hypotheses include retrograde degeneration from loss of cortical neurons or inflammation, amyloid and neurofibrillary tangles disrupting normal retinal cell function, and reduced vascularization. The retinal thickness (peripapillary RNFL and macular GC-IPL) was reported to be generally lower in MCI patients than in controls; however, the magnitude failed to reach a statistical significance in a meta-analysis ${ }^{7}$. The lack of statistical significance may be partly owing to a small number of eligible studies. Moreover, it has been hypothesized that activation of Müller cells and swelling of neurons may occur in the early stages of neurodegeneration, resulting in an increase in macular thickness ${ }^{18}$. In this study, we compared the thickness of all the layers of the retina between MCI patients and controls using automated segmentation software and found no significant differences between the two groups. Our results showed that significantly lower VD was observed in the MCI group than in the control group, although there was no significant 
difference in the retinal neuronal thickness. This suggests that changes in the retinal microvasculature precede detectable changes in retinal neuronal thickness, and thus, they could be earlier biomarkers.

We also demonstrated a correlation between retinal microvasculature and the thickness of each retinal layer in the eyes of MCI patients. A previous study reported a correlation between loss of retinal microvasculature in the DCP and GC-IPL thinning in the AD group ${ }^{11}$, whereas our results showed that VD of the SCP was correlated with GCL and IPL thickness. Considering that GCL-IPL is mainly supplied by the SCP, our results suggest that these retinal changes, namely GCL and IPL thinning and reduction of retinal microvasculature, are related to each other instead of being independent occurrences. Further exploration of this association is needed to elucidate this relationship, and it may improve our understanding of the dynamics of AD pathology in the nervous system, which has important implications for determining AD risk.

The $A P O E-\varepsilon 4$ is the most common genetic risk factor for $\mathrm{AD}$ and is linked to other neurodegenerative conditions that affect cognition. APOE is known to modulate multiple mechanistic pathways, including cholesterol/ lipid homeostasis, synaptic function, glucose metabolism, neurogenesis, tau phosphorylation, neuroinflammation, and aggregation of $\mathrm{A} \beta$ in the central nervous system. In addition, $A P O E$ genotypes differentially modulate the function of the cerebral vasculature, reduce cerebral blood flow, and increase blood-brain barrier leakage and cerebral amyloid angiopathy ${ }^{19}$. Reduced electroretinography responses and lower retinal and choroidal vascular endothelial growth factor were reported in $A P O E-\varepsilon 4$ mice $^{20,21}$. In our study, we showed that $A P O E-\varepsilon 4$ carriers had decreased VD of the DCP compared with non-carriers, suggesting that the APOE genotype causes changes in the microvasculature. Subsequent studies with a larger number of patients are needed.

This study had some limitations. The major limitation was the relatively small sample size, and this may have resulted in a lack of significant findings in some analyses. In particular, since the comparison of OCT and OCTA parameters between APOE- $\boldsymbol{\varepsilon} 4$ genotypes was an explorative analysis including a small number of eyes, we did not apply multiple testing adjustments. Further investigations with a large sample size are required. We only analyzed a single ethnic group (Korean) thus our results may not be generalizable to other populations. Comparisons among various ethnic groups would be interesting topics for future studies. Although there were no statistically significant differences, older age in the MCI group could have possibly influenced OCTA results. In this study, we analyzed MCI patients who were easily accessible in our clinical practice, but further longitudinal studies including preclinical $\mathrm{AD}, \mathrm{MCI}$, and $\mathrm{AD}$ participants will be needed to determine whether the evaluation of the retinal microvasculature using OCTA has value as an early biomarker in AD.

In conclusion, retinal microvasculature changes in patients with $\mathrm{MCI}$ owing to $\mathrm{AD}$ were detected using OCTA, and lower VD of the SCP was correlated with thinner GCL and IPL in these patients. This suggests that changes in the retinal microvasculature precede the reduction in the thickness of the retinal layers in patients with $\mathrm{MCI}$ owing to $\mathrm{AD}$. The APOE genotype may also affect changes in retinal microvasculature. Evaluation of the retinal microvasculature may be used as a potential biomarker to detect vascular changes in MCI owing to $\mathrm{AD}$ and could be a new imaging target for early diagnosis and management of AD.

\section{Methods}

This case-control study was approved by the institutional review board of Yonsei University College of Medicine (IRB approval number: 3-2018-0156) and was conducted in accordance with the tenets of the Declaration of Helsinki. All study participants provided informed consent. We recruited patients and controls from the Gangnam Severance Hospital between September 2017 and December 2018. Trained and qualified neurologists made the diagnosis of MCI owing to AD based on the National Institute on Aging-Alzheimer's Association criteria ${ }^{22}$. All MCI subjects tested positive for $\mathrm{A} \beta$ deposition in the brain on ${ }^{18} \mathrm{~F}$-florbetaben amyloid PET; brain magnetic resonance imaging and laboratory tests (e.g., thyroid function tests, serum vitamin B12, and folate levels) were performed to exclude other causes of cognitive decline. The MCI group underwent a neuropsychological test battery (MMSE, CDR, and $\mathrm{SNSB}^{23}$ ) for the assessment of global cognitive function. Genetic testing for APOE was performed only in patients who agreed to undergo the testing. The control group comprised of volunteers who were scheduled to undergo cataract surgery and who were clinically assessed as cognitively normal based on clinical interviews with patients and their caregivers. The control group subjects had no history of amnesia and no previous history of head trauma or neurological or psychiatric illness. We excluded subjects previously diagnosed with clinically apparent $\mathrm{AD}$, uncontrolled hypertension, or uncontrolled diabetes.

All subjects underwent corrected distance visual acuity (logMAR), intraocular pressure ( $\mathrm{mmHg}$ ), and spherical equivalent measurements. Detailed anterior segment and fundus examinations were performed, and widefield color fundus images were taken using a laser scanning ophthalmoscopy device (Optomap; Optos Plc., Dunfermline, UK). Examination of the microvasculature and retinal thickness of each layer of the macula was performed using two different devices, ZEISS OCTA and SPECTRALIS OCT, respectively, on the same day.

We performed OCTA (ZEISS HD-OCT Model 5000 instrument with AngioPlex, Carl Zeiss Meditec, Dublin, CA, USA) to examine the retinal microvasculature covering a macular area of $6 \times 6 \mathrm{~mm}$ centered on the fovea for the SCP and DCP of the retina. The projection artifacts of the superficial layer were removed in the deep-layer images using built-in software. All images were exported into the Image J 1.50 software (National Institutes of Health, Bethesda, MD, USA) to measure the FAZ area and VD. We manually outlined the FAZ using the polygon selection tool ${ }^{24,25}$ and calculated the VD of the $6 \times 6-\mathrm{mm}$ macula, except the FAZ (central foveal 0.5 -mm radius area), by image thresholding, binarization, and skeletonization according to the methods described in a previous study $^{26}$. Two independent researchers (EYC and JYS) obtained and evaluated OCTA findings, and the average values were used for the statistical analysis. In the analysis of the FAZ and VD in Image J, and manual edits of OCTA segmentation, researchers were masked to participant characteristics to avoid bias.

To examine the thickness of each retinal layer, we used SPECTRALIS OCT (Spectralis HRA + OCT; Heidelberg Engineering, Franklin, MA, USA) to scan a macular area of $6 \times 4 \mathrm{~mm}$ centered on the fovea. The retinal 
segmentation software accompanying the device was used to identify each retinal neuronal layer and quantify its thickness; the software automatically calculated the average retinal thickness of each retinal layer. For the analysis, retinal layers were divided into RNFL, GCL, IPL, inner nuclear layer, outer plexiform layer, outer nuclear layer, and retinal pigment epithelium (RPE).

We reviewed all segmentation images of OCTA and OCT and manually modified significant segmentation errors. We excluded eyes with retinal diseases (e.g., age-related macular degeneration, diabetic retinopathy, epiretinal membrane, and macular hole), optic nerve diseases (e.g., glaucoma, and ischemic optic neuropathy), significant media opacity with poor quality (signal strength $<70$ on OCTA and $<25$ on OCT), or high refractive error over $\pm 6 \mathrm{D}$ from the analysis. Thus, eight eyes from the MCI group (two eyes with epiretinal membrane, five eyes with drusen or RPE changes, one eye with low signal strength) and 25 eyes of controls (three eyes with epiretinal membrane, seven eyes with drusen or RPE changes, 15 eyes with low signal strength) were excluded from the analysis.

When analyzing OCT and OCTA images, we used the average values of the standard retinal subfields, namely central, superior, temporal, inferior, and nasal quadrants of the inner and outer rings as defined in the Early Treatment Diabetic Retinopathy Study ${ }^{27}$. The diameters of the central, inner, and outer rings were 1, 3, and $6 \mathrm{~mm}$, respectively.

Statistical analysis. For comparison of the baseline characteristics, the thickness of each retinal layer, and OCT parameters between the MCI group and controls, we used the Mann-Whitney test for continuous variables and Fisher's exact test for categorical variables. In the comparison of the VD of the SCP in each subfield between the MCI and controls groups, the Benjamini-Hochberg method was used for multiple testing adjustment. To assess the relationship between VD and retinal layer thickness, Spearman's correlation was used. Because the covered macular area differed in the two devices $(6 \times 6 \mathrm{~mm}$ in OCTA and $6 \times 4 \mathrm{~mm}$ in OCT), we used the average values of VD and retinal layer thickness in the inner ring in the analysis. We categorized the APOE genotype into $A P O E-\varepsilon 4$ carriers ( 10 eyes from 6 patients) and non-carriers (18 eyes from 10 patients) because of the low prevalence of $A P O E-\varepsilon 4$ homozygotes. We compared OCT and OCTA parameters between APOE- $\varepsilon 4$ carriers and noncarriers using the Mann-Whitney test for continuous variables and Fisher's exact test for categorical variables. Statistical analysis was performed using SPSS software 21 (SPSS, Inc., Chicago, IL, USA), and p-values $<0.05$ were considered statistically significant.

Received: 12 March 2020; Accepted: 19 November 2020

Published online: 19 January 2021

\section{References}

1. de la Torre, J. C. Alzheimer disease as a vascular disorder: nosological evidence. Stroke. 33, 1152-1162 (2002).

2. Gatz, M. et al. Role of genes and environments for explaining Alzheimer disease. Arch. Gen. Psychiatry. 63, 168-174 (2006).

3. Verghese, P. B., Castellano, J. M. \& Holtzman, D. M. Apolipoprotein E in Alzheimer's disease and other neurological disorders. Lancet Neurol. 10, 241-252 (2011).

4. Farrer, L. A. et al. Effects of age, sex, and ethnicity on the association between apolipoprotein E genotype and Alzheimer disease: a meta-analysis-APOE and Alzheimer Disease meta analysis consortium. JAMA 278, 1349-1356 (1997).

5. Salloway, S. et al. A phase 2 multiple ascending dose trial of bapineuzumab in mild to moderate Alzheimer disease. Neurology. 73 , 2061-2070 (2009).

6. Wright, C. F., Hall, A., Matthews, F. E. \& Brayne, C. Biomarkers, dementia, and public health. Ann. N. Y. Acad. Sci. 1180, 11-19 (2009).

7. Chan, V. T. T. et al. Spectral-domain OCT measurements in Alzheimer's disease: a systematic review and meta-analysis. Ophthalmology 126, 497-510 (2019).

8. Knoll, B. et al. Retinal nerve fiber layer thickness in amnestic mild cognitive impairment: case-control study and meta-analysis. Alzheimers Dement. 19, 85-93 (2016).

9. Ko, F. et al. Association of retinal nerve fiber layer thinning with current and future cognitive decline: a study using optical coherence tomography. JAMA Neurol. 75, 1198-1205 (2018).

10. Mutlu, U. et al. Association of retinal neurodegeneration on optical coherence tomography with dementia: a population-based study. JAMA Neurol. 75, 1256-1263 (2018).

11. Jiang, H. et al. Altered macular microvasculature in mild cognitive impairment and Alzheimer disease. J. Neuro-Ophthalmol. 38, 292-298 (2018).

12. Bulut, M. et al. Evaluation of optical coherence tomography angiographic findings in Alzheimer's type dementia. Br. J. Ophthalmol. 102, 233-237 (2018).

13. O’Bryhim, B. E., Apte, R. S., Kung, N., Coble, D. \& Van Stavern, G. P. Association of preclinical Alzheimer disease with optical coherence tomographic angiography findings. JAMA Ophthalmol. 136, 1242-1248 (2018).

14. Grewal, D. S., Polascik, B. W., Hoffmeyer, G. C. \& Fekrat, S. Assessment of differences in retinal microvasculature using OCT angiography in Alzheimer's disease: a twin discordance report. Ophthalmic. Surg. Lasers Imaging Retina. 49, 440-444 (2018).

15. Den Haan, J. et al. Retinal thickness correlates with parietal cortical atrophy in early-onset Alzheimer's disease and controls. Alzheimers Dement. 1, 49-55 (2017).

16. Bell, R. D. \& Zlokovic, B. V. Neurovascular mechanisms and blood-brain barrier disorder in Alzheimer's disease. Acta Neuropathol. 118, 103-113 (2009)

17. Claudio, L. Ultrastructural features of the blood-brain barrier in biopsy tissue from Alzheimer's disease patients. Acta Neuropathol. (Berl) 91, 6-14 (1996).

18. Ascaso, F. J. et al. Retinal alterations in mild cognitive impairment and Alzheimer's disease: an optical coherence tomography study. J. Neurol. 261, 1522-1530 (2014).

19. Tai, L. M. et al. The role of APOE in cerebrovascular dysfunction. Acta Neuropathol. 131, 709-723 (2016).

20. Antes, R. et al. ApoE4 induces synaptic and ERG impairments in the retina of young targeted replacement apoE4 mice. PLoS ONE 8, e64949 (2013).

21. Antes, R. et al. VEGF Mediates ApoE4-Induced Neovascularization and Synaptic Pathology in the Choroid and Retina. Curr. Alzheimer Res. 12, 323-334 (2015). 
22. Albert, M. S. et al. The diagnosis of mild cognitive impairment due to Alzheimer's disease: recommendations from the National Institute on Aging-Alzheimer's Association workgroups on diagnostic guidelines for Alzheimer's disease. Alzheimers Dement. 7, 270-279 (2011).

23. Ahn, H.-J. et al. Seoul neuropsychological screening battery-dementia version (SNSB-D): a useful tool for assessing and monitoring cognitive impairments in dementia patients. J. Korean Med. Sci. 25, 1071-1076 (2010).

24. Shahlaee, A., Pefkianaki, M., Hsu, J. \& Ho, A. C. Measurement of foveal avascular zone dimensions and its reliability in healthy eyes using optical coherence tomography angiography. Am. J. Ophthalmol. 161, 50-55 (2016).

25. Carnevali, A. et al. Optical coherence tomography angiography analysis of retinal vascular plexuses and choriocapillaris in patients with type 1 diabetes without diabetic retinopathy. Acta Diabetol. 54, 695-702 (2017).

26. Chidambara, L. et al. Characteristics and quantification of vascular changes in macular telangiectasia type 2 on optical coherence tomography angiography. Br. J. Ophthalmol. 100, 1482-1488 (2016).

27. Hee, M. R. et al. Topography of diabetic macular edema with optical coherence tomography. Ophthalmology 105, 360-370 (1998).

\section{Acknowledgements}

This study was supported by a faculty research grant from Yonsei University College of Medicine, 2017-32-0037 and a "Hankookilbo Myung-Ho Seung" faculty research grant from Yonsei University College of Medicine, 6-2020-0236 and 3-2020-0459. The authors declare that no competing interests exist with the funder. The funders had no role in study design, data collection and analysis, the decision to publish, or preparation of the manuscript.

\section{Author contributions}

Design and conduct of the study: J.Y.S., E.Y.C., M.K., H.K.L., and S.H.B. Collection, management, analysis, and interpretation of the data: J.Y.S., E.Y.C., M.K., H.K.L., and S.H.B. Preparation, review, or approval of the manuscript: J.Y.S., E.Y.C., M.K., H.K.L., and S.H.B. Decision to submit the manuscript for publication: J.Y.S., E.Y.C., M.K., H.K.L., and S.H.B.

\section{Competing interests}

The authors declare no competing interests.

\section{Additional information}

Correspondence and requests for materials should be addressed to M.K.

Reprints and permissions information is available at www.nature.com/reprints.

Publisher's note Springer Nature remains neutral with regard to jurisdictional claims in published maps and institutional affiliations.

(c) (i) Open Access This article is licensed under a Creative Commons Attribution 4.0 International License, which permits use, sharing, adaptation, distribution and reproduction in any medium or format, as long as you give appropriate credit to the original author(s) and the source, provide a link to the Creative Commons licence, and indicate if changes were made. The images or other third party material in this article are included in the article's Creative Commons licence, unless indicated otherwise in a credit line to the material. If material is not included in the article's Creative Commons licence and your intended use is not permitted by statutory regulation or exceeds the permitted use, you will need to obtain permission directly from the copyright holder. To view a copy of this licence, visit http://creativecommons.org/licenses/by/4.0/.

(c) The Author(s) 2021 